\title{
Income-Related Inequality in Life-Years and Quality-Adjusted Life-Years
}

\author{
Ulf-G. Gerdtham*ànd Magnus Johannesson \\ Stockholm School of Economics \\ This version: September 1999
}

\begin{abstract}
We estimate the income-related inequality in Sweden with respect to life-years and quality-adjusted life-years (QALYs). We use a large data-set from Sweden with over 40,000 individuals followed up for 10-16 years, to estimate the survival and quality-adjusted survival in different income groups. For both life-years and QALYs we discover inequalities in health favouring the higher income groups. For men (women) in the youngest age-group (20 - 29 years) the number of QALYs is 43.7 (45.7) in the lowest income decile and 47.2 (49.0) in the highest income decile.
\end{abstract}

Key words: Health inequality, income, mortality, QALYs.

JEL-classification: D30; I10; I12.

*Address for correspondence: Ulf-G. Gerdtham, Department of Economics, Stockholm School of Economics, Box 6501, S-113 83 Stockholm, Sweden. Tel: +46-8-7369283. Fax: +46-8-302115. E-mail: heug@hhs.se.

†We thank Eddy van Doorslaer for helpful comments. 


\section{Introduction}

The issue of income-related inequalities in health has received increasing attention in health economics in recent years (van Doorslaer et al. 1997; Kakwani et al. 1997; Gerdtham et al. 1999). Van Doorslaer et al. (1997) estimated income-related inequalities in health for nine industrialised countries, using the concentration index as the measure of inequality. Their results showed that the inequalitites in health significantly favoured the higher income groups in all countries (van Doorslaer et al. 1997). Self assessed overall health status was used as the measure of health in the study by van Doorslaer et al (1997). Health status is, however, an incomplete measure of health since it ignores the length of life. It would thus also be interesting to investigate income related inequalities with respect to length of life. That there is an association between income and mortality has been shown in many studies (Lutter and Morrall 1994; Viscusi 1994a,b; Keeney 1997). Several previous studies have focused on inequalities in mortality (Le Grand 1987, 1989; Kunst and Mackenbach 1992,1994; Valkonen 1989), but they have not measured the concentration index of income-related inequality.

Ideally one would like to use a health measure that incorporates both health status and length of life in one measure. Such a health measure, quality-adjusted life-years (QALYs), has been developed in the field of economic evaluation of health care (Drummond et al. 1997). QALYs are constructed by weighting life-years between 0 (dead) and 1 (full health) depending on the health status. Gerdtham et al. (1999) calculated health concentration indices for QALY weights as a measure of health status, but the number of QALYs has not yet been used in studies on income-related inequality. 
The purpose of this study is twofold. The first purpose is to develop methods for using life-years and QALYs as the health measure in studies of income-related inequalities in health. The second purpose is to report empirical results for Sweden. Our methods are described below, followed by a presentation of the results. The paper ends with some concluding remarks.

\section{Methods}

\subsection{Income-related inequality in life-years}

To estimate how the life expectancy varies with income we use data from Statistic Sweden's Survey of Living Conditions (the ULF survey) (Statistics Sweden 1997) which have been linked to survival data from the National Causes of Death Statistics and to income data from the National Income Tax Statistics. Every year, Statistics Sweden conducts systematic surveys of living conditions, in the form of one-hour personal interviews with randomly selected adults aged 16-84 years. Since 1975 about 7,000 individuals have been interviewed each year. We use pooled data from the annual interviews conducted in 1980 - 1986 for all the subjects aged $20-84$ years at the time of the interview. We exclude subjects younger than 20 years of age, to avoid having subjects who are still in high school (most people in Sweden finish high school when they are 19). The total sample consists of 43, 898 individuals. After correcting for missing values, the sample is reduced to 43, 366 individuals. The mortality experience of the sample is recorded until December 31, 1996. Table 1 gives summary statistics for the variables we use in the regression analysis (Table 1 in here).

A Cox proportional hazard regression is estimated on the relationship between 
mortality and income, controlling for age and gender (Cox 1972). The dependent variable is the survival time in years and the survival status at the end of followup. The date of death is recorded for all subjects who had died by December 31, 1996. The survival time is estimated as the number of years from the interview date to the date of death. The censored survival time of persons alive at the end of 1996 is estimated as the number of years from the interview date to December 31, 1996. The average censored survival time in the sample is 12.31 years, and $80 \%$ are alive at the end of follow-up on December 31, 1996.

The Cox regression makes no distributional assumptions about the functional form of the baseline hazard, as required for most other hazard functions. Besides depending on time, the hazard rate will depend on individual characteristics (covariates). Taking these into account, the hazard rate at time $t$ for individual $i$ may be written:

$$
h(t ; X)=h(t, 0) \cdot e^{X_{i} \beta}
$$

$h(t, 0)$ is the unknown baseline hazard rate at time $t$ (the hazard rate for the respective individual when all covariate values are equal to zero), $X$ is a vector of covariates which shifts the hazard function proportionally, and $\beta$ is a vector of parameters. While no assumptions are made about the functional form of the baseline hazard, the Cox model assumes a proportional relationship between the hazard function and the log-linear function of the covariates, i.e. it is assumed that the ratio of the hazard function for two individuals does not depend on time, i.e. $e^{X_{i} \beta}=h(t ; X) / h(t, 0)$ measures the hazard function at time $t$ for an individual with a vector of covariates $X$ relative to the baseline function for an individual with covariate vector 0 . 
As an independent variable in the regression analysis we use the annuitized lifetime household income per consumption unit in 1996 prices in Swedish Crowns (SEK) (Exchange rate 1996: $\$ 1=$ SEK 6.71). This income measure consists of two components that are added together: the annuity of the life-time disposable income and the annuity of net wealth. In our data set we have information about the disposable income of the household in the interview year. Disposable income is converted to 1996 prices using the consumer price index. A problem with using the disposable income in a single year is that it may be an imperfect measure of the annual consumption in our observation period. The income varies between years and individuals may borrow or save to even out the consumption between periods. We therefore estimate the expected life-time disposable income. The lifetime disposable income is estimated based on the percentage change in income with age in our sample. The percentage change in income with age is estimated separately for men and women and for the three education categories in our data set. $^{1}$ To estimate the life-time income, the average life expectancy for men and women in Sweden is used (Statistics Sweden 1998). A 3\% discount rate is used to estimate the lifetime disposable income in present value terms. The annuity of the life-time income is then used in the analysis.

From the National Income Tax Statistics we also have information about the taxable net wealth (total taxable assets minus total liabilities) of the household

\footnotetext{
${ }^{1} \mathrm{~A}$ regression equation of disposable income as a function of age and age-squared is used to estimate the percentage change in disposable income for each year until the age of 65 (the retirement age). After retirement the annual income is assumed to be constant; to estimate the percentage change in income at retirement we use the the average income in the $65-69$ years age-group in our data (for the gender and education category of the individual).
} 
during the interview year. Taxable net wealth is converted to 1996 prices using the consumer price index. The data recorded in the National Income Tax Statistics is the taxable net wealth that is used as the basis for the tax on wealth in Sweden. Due to the tax rules, not all assets are valued at the market value in the taxable net wealth measure. Thus the measure systematically undervalues net wealth. The measure also depends on the tax rules at the time of the survey, which means that the values may not be strictly comparable between the different interview years. We therefore carry out an approximation of the net wealth at market value. ${ }^{2}$ The annuity of net wealth is then added to the annuity of annual disposable income to obtain our income measure.

To find the optimal functional relationship between income and the mortality rate a Box-Cox analysis is carried out (Box-Cox 1964), i.e. a Box-Cox transformation parameter $\lambda$ as defined by the operator: $X(\lambda)=\left(X^{\lambda}-1\right) / \lambda$ for $\lambda \neq 0$ or $\ln X$ for $\lambda=0$, where $X$ is income. In this procedure we determine the maximum likelihood point estimate for $\lambda$ where a one-dimensional grid search is carried out over the interval -1 to 1 for $\lambda$ at 0.05 increments. In the regression we also control for age, age-squared and gender. The log-likelihood function is maximized at $\lambda=0.24$. In the appendix, Table A1, we show the estimated hazard function

\footnotetext{
${ }^{2}$ To carry out this approximation we estimate the ratio between net wealth at market value and taxable net wealth for each interview year. We then multiply the taxable net wealth by this ratio for each person to get the net wealth of each person. As the measure of net wealth at market value we use the average value in an investigation about the average household net wealth at market value in the Swedish adult population in 1983, 1984, 1985 and 1988 (Statistics Sweden 1990). The value of the household net wealth at market value was SEK 322, 938 in 1996 prices. The ratio between this value and the taxable net wealth was: 1.83 in 1980, 1.32 in 1981, 1.35 in 1982, 1.32 in 1983, 1.45 in 1984, 1.47 in 1985, and 1.38 in 1986.
} 
where $\lambda$ is set at 0.24 . The estimated hazard function is then used to estimate the life-expectancy of all subjects in our sample. The hazard function gives the annual mortality risk and life expectancy is estimated by estimating the annual risk at all ages for an individual. We estimate the life expectancy in seven agegroups ( $20-29$ years, $30-39$ years, $40-49$ years, $50-59$ years, $60-69$ years, $70-79$ years, $80-84$ years). To standardise for age within each age-group the age of all individuals in an age-group is set to the mean age in that age-group. ${ }^{3}$ The estimated life expectancy for all individuals in our sample is used to calculate the concentration index for life-years in the different age-groups and for the entire sample (estimated as the weighted average of the concentration indices in the different age-groups). The concentration index plots the cumulative proportion of a measure of health against the cumulative proportion of the population ranked from the lowest to highest in terms of income. The concentration index is defined as twice the area between the concentration curve and the diagonal which represents an equal distribution of health across the population, and can take values between +1 and -1 . A positive concentration index signifies income-related health inequality; it is +1 when all health is concentrated in those individuals in the highest income group.

The calculations are carried out for men and women separately. The calculations are also carried out with and without discounting of life-years. In economic evaluations it is common to discount life-years and in the analysis with discounting of life-years we use the currently recommended discount rate of $3 \%$ (Weinstein

\footnotetext{
${ }^{3}$ For both men and women, the following mean age figures are used for $20-29$ years, $30-39$ years, $40-49$ years, $50-59$ years, $60-69$ years, $70-79$ years and $80-84$ years: 24.45 years, 34.61 years, 44.12 years, 54.53 years, 64.40 years, 74.37 years and 81.73 years.
} 
et al. 1996).

It should be noted that the concentration indices calculated with respect to life-years are not directly comparable to the indices estimated by van Doorslaer et al. (1997). This is because the concentration indices in van Doorslaer et al (1997) were defined with respect to ill-health rather than health as here. It is impossible to estimate a concentration index with respect to ill-health for lifeyears unless some upper limit of life-years is defined so that the loss of life-years can be estimated relative to this upper limit.

\subsection{Income-related inequality in quality-adjusted life-years (QALYs)}

We also adjust the life-years for quality of life in order to be able to estimate the income-related inequality in QALYs. No data about QALY weights is collected in the ULF survey. However, we assigned QALY weights to all subjects in our database, based on a categorical measure of the overall health status that was included in the ULF survey. In the categorical health rating question the individuals rated their own current health status on a three-point scale (poor health, fair health, good health). This type of categorical health measure has been shown to capture important information about the individual's health and to be an important predictor of mortality (Kaplan and Camacho 1983; Connelly et al. 1989; Idler and Kasl 1991; Wannamethe and Shaper 1991).

To estimate the QALY weights for poor health, fair health and good health we use another data set collected in Uppsala County in Sweden (Gerdtham et al. 1999; Lundberg et al. 1999). This study included a measurement of QALY weights by the time trade-off method. It also included a categorical five-point 
health rating question (poor health, fair health, good health, very good health, excellent health). This five-point question is collapsed into a three-point scale to correspond to the question used in the ULF survey. ${ }^{4}$

An OLS regression with the QALY weight as dependent variable and the three-point health rating question as independent variable is estimated to predict the QALY weight for poor health, fair health and good health. Age and gender are also included in this regression analysis since these variables may affect the QALY weight independently of the rating on the categorical health rating question (Lundberg et al. 1999). ${ }^{5}$ This regression equation is used to predict a QALY weight for all subjects in the ULF survey based on their age, gender and rating of their current health status. Based on these QALY weights we calculate concentration indices for the income-related inequality in health status for men and women in different age groups. These indices are calculated with respect to both health (i.e. the QALY weight) and ill-health (1-QALY weight), so that the results for ill-health can be compared to the results in van Doorslaer et al. (1997).

To estimate the expected number of QALYs for an individual in our database it is not sufficient to know the current QALY weight, but the QALY weight in future years is needed as well. To predict the change in the QALY weight over time for the individuals in our database we estimate a regresion equation with the

\footnotetext{
${ }^{4}$ Excellent health, very good health and good health is assumed to correspond to good health in the three-point scale used in the ULF survey.

${ }^{5} \mathrm{QALY}=0.683+0.261 \cdot$ GOOD HEALTH + 0.143·FAIR HEALTH + 0.009521-MALE + 0.005669-AGE2 - 0.000649-AGE3 - 0.000971·AGE4 - 0.0411·AGE5 - 0.163·AGE6 - 0.261·AGE7;

AGE2 $=30-39$ years, AGE3 $=40-49$ years, AGE4 $=50-59$ years, AGE5 $=60-69$ years, AGE $6=70-79$ years and AGE7 $=80-84$ years (Baseline $=20-29$ years).
} 
predicted QALY weight as the dependent variable and age, age-squared, gender and income as independent variables. To find the optimal functional relationship between income and the QALY weight we again use a Box-Cox analysis. The $\log$-likelihood function is maximized at $\lambda=0.35$. In the Appendix, Table A2, we show the estimated regression where $\lambda$ is set at 0.35 . An OLS regression equation is used with a logistic transformation of the dependent variable since the QALY weights are between 0 and 1 (Greene 1997). ${ }^{6}$ Based on this regression we predict the change in the QALY weights over time for the subjects in our database. The information about the QALY weights over time is combined with the estimated life-years to estimate the expected number of QALYs for all subjects in our database. The estimated number of QALYs for all individuals in our sample is used to calculate the concentration index for QALYs in the different age-groups and for the entire sample (estimated as the weighted average of the concentration indices in the different age-groups). The calculations are carried out for men and women separately and with and without discounting of QALYs. As noted above, these concentration indices for QALYs are not directly comparable to the indices calculated by van Doorslaer et al. (1997), since the indices are calculated with respect to health rather than ill-health.

\footnotetext{
${ }^{6} \mathrm{~A}$ logistic transformation of the dependent variable could not be used for the equation where we predict QALY weights based on the self rated health status using data from the Uppsala study. This is because a large proportion of the QALY weights in that data set are equal to 1 (full health). However, all the predictions from that equation for our sample in the ULF database fall between 0 and 1 .
} 


\section{Results}

\subsection{Income-related inequality in remaining life-years}

The estimated life expectancy in different age-groups are shown in Tables 2 (men) and 3 (women) (Tables 2 and 3 in here). The life expectancy is shown for each income decile in each age-group. The tables show that life expectancy is distributed unequally between income groups, favouring higher income groups. For men in the age-group $20-29$ years the life expectancy is 49.7 years (25.9 with discounting) in the lowest income decile and 53.8 years (26.8 with discounting) in the highest income decile. For men in the age-group $80-84$ years the life expectancy is 4.6 years (4.2 with discounting) in the lowest income decile and 6.6 years (5.9 with discounting) in the highest income decile. The pattern is similar for women. For women in the age-group $20-29$ years the life expectancy is 54.7 years (27.0 with discounting) in the lowest income decile and 58.5 years $(27.8$ with discounting) in the highest income decile. For women in the age-group $80-84$ years the life expectancy is 6.6 years (5.8 with discounting) in the lowest income decile and 8.6 years (7.4 with discounting) in the highest income decile.

The concentration indices are also shown in Tables 2-3. For men the concentration index increases with age from 0.0112 in the youngest age-group to 0.0589 in the oldest age-group. The pattern with respect to age is similar with discounting, although the indices decrease somewhat to 0.0052 in the youngest age-group and 0.0534 in the oldest age-group. The weighted average for all age-groups is 0.0203 without discounting and 0.0146 with discounting. For women the concentration index also increases with age from 0.0098 in the youngest age-group to 0.0426 in the oldest age-group. With discounting the concentration indices de- 
crease to 0.0041 in the youngest age-group and 0.0376 in the oldest age-group. The weighted average for all age-groups is 0.0171 without discounting and 0.0118 with discounting.

\subsection{Income-related inequality in health status}

In Tables 4 (men) and 5 (women) the QALY weights are shown for the different age-groups and income deciles. The results are consistent with the results for life-years, with the distribution of health status with respect to income favouring higher income groups. For men in the age-group $20-29$ years the QALY weight is 0.94 in the lowest income decile and 0.95 in the highest income decile. For men in the age-group $80-84$ years the QALY weight is 0.59 in the lowest income decile and 0.61 in the highest income decile. For women in the age-group $20-29$ years the QALY weight is 0.92 in the lowest income decile and 0.94 in the highest income decile. For women in the age-group $80-84$ years the QALY weight is 0.55 years in the lowest income decile and 0.59 in the highest income decile.

The concentration indices for health status (the QALY weights) are also shown in Tables 4-5. For men the concentration index increases with age until the oldest age-group and varies between 0.0015 in the youngest age-group and 0.0126 in the $70-79$ years age-group. The weighted average for all age-groups is 0.0060 . For women the concentration index increases with age from 0.0022 in the youngest age-group to 0.0111 in the oldest age-group. The weighted average for all agegroups is 0.0058 . For comparisons with previous studies the concentration indices for ill health (1-QALY weight) are also shown in Tables 4-5. These indices show a different pattern with respect to age. For both men and women the indices in 
absolute terms increase with age until the middle age-group (50 - 59 years) and then decrease again. The weighted average concentration index for ill-health is -0.0477 for men and -0.0381 for women. These figures are similar to the figure of -0.0347 for Sweden reported in the study by van Doorslaer et al. (1997).

\subsection{Income-related inequality in remaining QALYs}

The estimated number of remaining QALYs in different age-groups and income deciles are shown in Tables 6 (men) and 7 (women) (Tables 6 and 7 in here). The pattern is the same as for life-years. For men in the age-group $20-29$ years the number of QALYs is 43.7 (23.5 with discounting) in the lowest income decile and 47.2 (24.5 with discounting) in the highest income decile. For men in the age-group $80-84$ years the number of QALYs is 2.3 (2.1 with discounting) in the lowest income decile and 3.3 (3.0 with discounting) in the highest income decile. For women in the age-group $20-29$ years the number of QALYs is 45.7 (23.8 with discounting) in the lowest income decile and 49.0 (24.8 with discounting) in the highest income decile. For women in the age-group $80-84$ years the number of QALYs is 2.9 (2.6 with discounting) in the lowest income decile and 3.9 (3.4 with discounting) in the highest income decile.

The concentration indices for QALYs are also shown in Tables 6-7. The concentration indices increase somewhat for both men and women compared to the case for life-years. The weighted average for all age-groups for men is 0.0251 without discounting of QALYs and 0.0201 with discounting of QALYs. For women the weighted average for all age-groups is 0.0213 without discounting and 0.0170 with discounting. 


\section{Concluding remarks}

We have estimated the income-related inequality in Sweden with respect to lifeyears, health status (current QALY weights) and QALYs. For all measures we discovered inequalities in health favouring the higher income groups.

As a measure of the income-related inequality in health we used the concentration index as used previously by van Doorslaer et al. (1997) for health status. It is important to note, however, that our calculated concentration indices for life-years and QALYs are not directly comparable to the concentration indices in the study by van Doorslaer et al. (1997). This is because they calculated concentration indices with respect to ill-health rather than health. For life-years and QALYs it is not possible to define a measure of ill-health unless some upper limit of life-years is determined. However, for health status we calculated the concentration indices with respect to both ill-health and health. Our concentration index for ill-health was -0.0477 for men and -0.0381 for women, which is similar to the index of -0.0347 for Sweden in the study by van Doorslaer et al. (1997).

For men the difference in the number of life-years between the highest and the lowest income decile was 4.1 years (1.0 with discounting) in the youngest agegroup (20 - 29 years) and 2.1 years (1.7 with discounting) in the oldest age-group (80 - 84 years). After incorporation of quality of life these differences decreased to 3.6 QALYs (1.0 with discounting) in the youngest age-group and 1.0 QALYs (0.9 with discounting) in the oldest age-group. For women the corresponding differences in life-years and QALYs between the lowest and highest income deciles were 3.8 life-years (0.8 with discounting) and 3.4 QALYs (1.0 with discounting) in the youngest age-group and 2.1 life-years (1.6 with discounting) and 1.0 QALYs 
(0.9 with discounting) in the oldest age-group. The quality adjustment has two effects on the inequality in health that work in opposite directions. The first effect is that quality adjustment increases inequality since the quality weights tend to increase with income. The second effect is that quality adjustment decreases inequality since the additional life-years in higher income groups are not in full health. Overall the quality adjustment decreases the absolute differences between income deciles, but it leads to a slight increase in the concentration indices.

Our concentration indices for income-related inequalities tend to increase with age. This means that comparisons between for instance countries may be affected by differences in age distributions across countries. This is also true for the concentration indices calculated with respect to ill-health in the study by van Doorslaer et al. (1997). It is also interesting to note that the pattern with respect to age differs according to whether the concentration indices are defined in terms of ill-health or health. Our concentration indices with respect to ill-health (1QALY weight) for both men and women increase with age until 50-59 years and then decrease with age. On the other hand the corresponding indices for health (QALY weight) generally increase with age. The reason for these divergent results is that a specific absolute difference in health may imply a different relative difference depending on whether health or ill-health is being used. This also implies that the ranking in terms of income-related inequality in health between for instance countries may differ depending on whether ill-health or health is used in the calculated concentration indices.

Whether the observed differences in life-years and QALYs between income groups for Sweden should be considered as large and important or small and 
insignificant is a difficult issue. In relative terms the differences between income groups are rather small, with the exception of the oldest age-groups. For instance, in the youngest age-group for women the number of QALYs is only $7 \%$ higher in the highest income decile than in the lowest income decile. With discounting of QALYs this relative difference decreases to $4 \%$. However, with a valuation of a discounted QALY gained of $\$ 100,000$, as used in a recent study, even small differences in the number of QALYs would amount to important differences in welfare (Cutler and Richardson 1998). With such a value of QALY gained, the difference of 1.0 discounted QALYs between the highest and the lowest income decile for women in the youngest age-group would be equivalent to a difference of $\$ 100,000$ in life-time wealth.

Finally, we note that the relationship between income and health should not be interpreted causally. The purpose of this type of analysis is not to estimate the causal effect of income on health but merely to describe how health varies between income groups. The observed association between income and health can emerge both because income affects health and the other way around (for a review of this issue, see Smith (1999)). It could also be because income is correlated with other factors that affect health such as education, unemployment and the rate of time preference.

\section{References}

Box, G.E.P and D.R Cox, 1964, An analysis of transformations, Journal of the Royal Statistical Society, Ser B, 211-243.

Cox, D., 1972, Regression models and life tables (with discussion), Journal of the Royal Sta- 
tistical Society $74,187-220$.

Cutler, D. and E. Richardson, 1998, The value of health: 1970-1990, American Economic Review Papers and Proceedings 88, 97-100.

Drummond, M.F., O'Brien, B., Stoddart, G.L. and G.W. Torrance, 1997, Methods for the economic evaluation of health care programmes, second edition (Oxford Medical Publications, Oxford).

Gerdtham U.-G., Johannesson, M., Lundberg, L. and D. Isacson, 1999, A note on validating Wagstaff and van Doorslaer's health measure in the analysis of inequalities in health, Journal of Health Economics 18, 117-124.

Greene, W.H., 1997, Econometric analysis, third edition (Prentice-Hall, Upper Saddle River, New Jersey).

Idler, E.L. and S. Kasl, 1991, Health perceptions and survival: do global evaluations of health status really predict mortality?, Journal of Gerontology 46, 555-565.

Kakwani, N.C., Wagstaff, A. and E. van Doorslaer, 1997, Socioeconomic inequalities in health: measurement, computation and statistical inference, Journal of Econometrics 77, 87-103.

Kaplan, G.A. and T. Camacho, 1983, Perceived health and mortality: a nine year followup of the human population laboratory cohort, American Journal of Epidemiology 117, 292-304.

Keeney, R.L., 1997, Estimating fatalities induced by the economic costs of regulations, Journal of Risk and Uncertainty 14, 5-23. 
Kunst, A.E. and J.P. Mackenbach, 1992, An international comparison of socioeconomic inequalities in mortality, Report MGZ 92.11, Department of Public Health and Social Medicine, Erasmus University, the Netherlands.

Kunst, A.E. and J.P. Mackenbach, 1994, Size of mortality differences associated with educational level in nine industrialised countries, American Journal of Public Health 84, 932-937.

Le Grand, J., 1987, Inequality in health: some international comparisons, European Economic Review 31, 182-191.

Le Grand, J., 1989, An international comparison of distributions of ages-at-death, in: J. Fox, ed., Health inequality in European countries (Gower, Aldershot).

Lundberg, L., Johannesson, M., Isacson, D.G.L. and L. Borgquist, 1999, The relationship between health-state utilities and the SF-12 in a general population, Medical Decision Making 19, 128-140.

Lutter, R. and J.F. Morrall III, 1994, Health-health analysis: a new way to evaluate health and safety regulation, Journal of Risk and Uncertainty 8, 43-66.

Smith, J.P., 1999, Healthy bodies and thick wallets: the dual relation between health and economic status, Journal of Economic Perspectives 13, 145-166.

Statistics Sweden, 1997, Living conditions and inequality in Sweden: a 20 year perspective 1975-1995, Living conditions, Report 91 (Statistics Sweden, Stockholm).

Valkonen, T., 1989, Adult mortality and the level of education, in: J. Fox, ed., Health inequality in European countries (Gower, Aldershot). 
van Doorslaer, E., Wagstaff, A., Bleichrodt, H., Calonge, S., Gerdtham, U.-G., Gerfin, M., Geurts, J., Gross, L., Häkkinen, U., Leu, R.E., O’Donnell, O., Propper, C., Puffer, F., Rodriguez, M., Sundberg, G., and O. Winkelhake, 1997, Income-related inequalities in health: some international comparisons, Journal of Health Economics 16, 93-112.

Viscusi, W.K., 1994a, Risk-risk analysis, Journal of Risk and Uncertainty 8, 5-17.

Viscusi, W.K., 1994b, Mortality effects of regulatory costs and policy evaluation criteria. RAND Journal of Economics 25, 94-109.

Wannamethe, G. and A.G. Shaper, 1991, Self-assessment of health status and mortality in middle-aged British men, International Journal of Epidemiology 20, 239-245.

Weinstein, M.C., Siegel, J.E., Gold. M.R., Kamlet, K.S. and L.B. Russel, 1996, Recommendations of the Panel on Cost-Effectiveness in Health and Medicine, Journal of the American Medical Association 276, 1253-1258. 


\section{TABLES}

Table 1: Sample descriptives (number of observations=43,366).

Variable

Mean

Std

Survival:

Survival time ${ }^{a}$

12.31

3.58

Proportion alive at the end of follow-up

$0.80 \quad 0.40$

\section{Self-assessed health status:}

Proportion in "poor health"

0.07

0.25

Proportion in "fair health"

0.20

0.40

Proportion in "good health"

0.73

0.44

\section{Socioeconomic variables:}

Proportion men

Age

Annuitized life-time household income per consumption unit ${ }^{\mathrm{b}} 122101.80$

${ }^{a}$ The number of life-years from inclusion in the study to the end of follow-up.

${ }^{b}$ Life-time income is estimated as the sum of household net wealth and the life-time household disposable income. The estimate is in 1996 Swedish Crowns (SEK), exchange rate 1996 \$1=SEK 6.71. 
Table 2: Remaining life-years for males in different ages and income deciles. In parentheses we provide discounted values.

Income

\section{Remaining Life-Years}

Age groups

Deciles

\begin{tabular}{lccccccc}
\hline & $\mathbf{2 0 - 2 9}$ & $\mathbf{3 0 - 3 9}$ & $\mathbf{4 0 - 4 9}$ & $\mathbf{5 0 - 5 9}$ & $\mathbf{6 0 - 6 9}$ & $\mathbf{7 0 - 7 9}$ & $\mathbf{8 0 - 8 4}$ \\
INC1 & 49.685 & 40.126 & 31.099 & 22.075 & 14.634 & 8.130 & 4.554 \\
& $(25.851)$ & $(23.127)$ & $(19.909)$ & $(15.753)$ & $(11.496)$ & $(7.006)$ & $(4.158)$ \\
INC2 & 51.327 & 41.090 & 32.027 & 22.897 & 15.146 & 8.416 & 4.793 \\
& $(26.259)$ & $(23.487)$ & $(20.290)$ & $(16.181)$ & $(11.820)$ & $(7.223)$ & $(4.359)$ \\
INC3 & 51.805 & 41.409 & 32.345 & 23.227 & 15.362 & 8.559 & 4.917 \\
& $(26.372)$ & $(23.587)$ & $(20.417)$ & $(16.348)$ & $(11.954)$ & $(7.330)$ & $(4.463)$ \\
INC4 & 52.099 & 41.649 & 32.574 & 23.458 & 15.546 & 8.685 & 5.034 \\
& $(26.440)$ & $(23.661)$ & $(20.508)$ & $(16.465)$ & $(12.068)$ & $(7.424)$ & $(4.560)$ \\
INC5 & 52.332 & 41.863 & 32.773 & 23.650 & 15.715 & 8.818 & 5.146 \\
& $(26.494)$ & $(23.727)$ & $(20.586)$ & $(16.561)$ & $(12.173)$ & $(7.522)$ & $(4.652)$ \\
INC6 & 52.545 & 42.083 & 32.974 & 23.829 & 15.900 & 8.950 & 5.272 \\
& $(26.543)$ & $(23.795)$ & $(20.665)$ & $(16.650)$ & $(12.286)$ & $(7.619)$ & $(4.756)$ \\
INC7 & 52.728 & 42.305 & 33.176 & 24.008 & 16.077 & 9.100 & 5.413 \\
& $(26.585)$ & $(23.862)$ & $(20.744)$ & $(16.739)$ & $(12.394)$ & $(7.730)$ & $(4.872)$ \\
INC8 & 52.928 & 42.553 & 33.402 & 24.229 & 16.301 & 9.288 & 5.565 \\
& $(26.630)$ & $(23.937)$ & $(20.831)$ & $(16.848)$ & $(12.530)$ & $(7.868)$ & $(4.996)$ \\
INC9 & 53.169 & 42.888 & 33.686 & 24.503 & 16.610 & 9.553 & 5.850 \\
& $(26.685)$ & $(24.038)$ & $(20.941)$ & $(16.983)$ & $(12.717)$ & $(8.061)$ & $(5.227)$ \\
INC10 & 53.753 & 43.661 & 34.422 & 25.175 & 17.385 & 10.361 & 6.639 \\
& $(26.813)$ & $(24.264)$ & $(21.218)$ & $(17.306)$ & $(13.174)$ & $(8.637)$ & $(5.853)$ \\
\hline ALL & 52.237 & 41.963 & 32.848 & 23.705 & 15.868 & 8.986 & 5.319 \\
& $(26.467)$ & $(23.753)$ & $(20.611)$ & $(16.584)$ & $(12.261)$ & $(7.642)$ & $(4.790)$ \\
& & & & & & & \\
\hline $\mathbf{N}^{\mathrm{a}}$ & $\mathbf{4 0 7 8}$ & $\mathbf{4 4 8 7}$ & $\mathbf{3 4 7 4}$ & $\mathbf{2 9 9 4}$ & $\mathbf{3 0 5 3}$ & $\mathbf{2 5 0 7}$ & $\mathbf{7 2 9}$ \\
$\mathbf{C l}^{\mathbf{b}}$ & $\mathbf{0 . 0 1 1 2}$ & $\mathbf{0 . 0 1 2 7}$ & $\mathbf{0 . 0 1 5 2}$ & $\mathbf{0 . 0 1 9 8}$ & $\mathbf{0 . 0 2 6 7}$ & $\mathbf{0 . 0 3 7 5}$ & $\mathbf{0 . 0 5 8 9}$ \\
$\mathbf{C i d}^{\mathbf{c}}$ & $\mathbf{0 . 0 0 5 2}$ & $\mathbf{0 . 0 0 6 9}$ & $\mathbf{0 . 0 0 9 5}$ & $\mathbf{0 . 0 1 4 1}$ & $\mathbf{0 . 0 2 1 1}$ & $\mathbf{0 . 0 3 2 4}$ & $\mathbf{0 . 0 5 3 4}$
\end{tabular}

${ }^{a}$ Number of observations.

${ }^{\mathrm{b}}$ Concentration index of income-related inequalities in remaining life-years, undiscounted figures.

${ }^{c}$ Concentration index of income-related inequalities in remaining life-years, discounted figures. 
Table 3: Remaining life-years for females in different ages and income deciles. In parentheses we provide discounted values.

Income

\section{Remaining Life-Years}

Age groups

Deciles

\begin{tabular}{lccccccc}
\hline & $\mathbf{2 0 - 2 9}$ & $\mathbf{3 0 - 3 9}$ & $\mathbf{4 0 - 4 9}$ & $\mathbf{5 0 - 5 9}$ & $\mathbf{6 0 - 6 9}$ & $\mathbf{7 0 - 7 9}$ & $\mathbf{8 0 - 8 4}$ \\
INC1 & 54.692 & 44.851 & 35.694 & 26.321 & 18.282 & 10.906 & 6.561 \\
& $(27.014)$ & $(24.604)$ & $(21.686)$ & $(17.883)$ & $(13.698)$ & $(9.026)$ & $(5.796)$ \\
INC2 & 55.965 & 45.710 & 36.615 & 27.119 & 18.712 & 11.182 & 6.799 \\
& $(27.289)$ & $(24.850)$ & $(22.021)$ & $(18.295)$ & $(13.946)$ & $(9.219)$ & $(5.984)$ \\
INC3 & 56.341 & 45.998 & 36.909 & 27.449 & 18.924 & 11.316 & 6.923 \\
& $(27.367)$ & $(24.929)$ & $(22.125)$ & $(18.490)$ & $(14.066)$ & $(9.312)$ & $(6.082)$ \\
INC4 & 56.615 & 46.198 & 37.133 & 27.686 & 19.105 & 11.435 & 7.020 \\
& $(27.423)$ & $(24.984)$ & $(22.203)$ & $(18.610)$ & $(14.168)$ & $(9.394)$ & $(6.157)$ \\
INC5 & 56.847 & 46.382 & 37.318 & 27.876 & 19.291 & 11.545 & 7.143 \\
& $(27.470)$ & $(25.034)$ & $(22.268)$ & $(18.705)$ & $(14.273)$ & $(9.470)$ & $(6.254)$ \\
INC6 & 57.054 & 46.558 & 37.493 & 28.061 & 19.470 & 11.657 & 7.271 \\
& $(27.512)$ & $(25.082)$ & $(22.268)$ & $(18.785)$ & $(14.373)$ & $(9.547)$ & $(6.353)$ \\
INC7 & 57.252 & 46.751 & 37.679 & 28.261 & 19.656 & 11.792 & 7.402 \\
& $(27.552)$ & $(25.134)$ & $(22.268)$ & $(18.863)$ & $(14.477)$ & $(9.639)$ & $(6.454)$ \\
INC8 & 57.481 & 46.977 & 37.908 & 28.465 & 19.896 & 11.977 & 7.573 \\
& $(27.598)$ & $(25.194)$ & $(22.268)$ & $(18.940)$ & $(14.609)$ & $(9.766)$ & $(6.587)$ \\
INC9 & 57.774 & 47.284 & 38.200 & 28.733 & 20.187 & 12.242 & 7.837 \\
& $(27.656)$ & $(25.276)$ & $(22.571)$ & $(19.037)$ & $(14.769)$ & $(9.945)$ & $(6.788)$ \\
INC10 & 58.452 & 48.066 & 38.878 & 29.548 & 21.056 & 13.076 & 8.649 \\
& $(27.786)$ & $(25.480)$ & $(22.798)$ & $(19.356)$ & $(15.235)$ & $(10.499)$ & $(7.397)$ \\
\hline ALL & 56.847 & 46.477 & 37.383 & 27.952 & 19.458 & 11.713 & 7.317 \\
& $(27.467)$ & $(25.057)$ & $(22.287)$ & $(18.589)$ & $(14.361)$ & $(9.582)$ & $(6.385)$ \\
& & & & & & & \\
\hline $\mathbf{N}^{\mathrm{a}}$ & $\mathbf{3 9 0 9}$ & $\mathbf{4 4 0 9}$ & $\mathbf{3 4 1 7}$ & $\mathbf{3 0 1 6}$ & $\mathbf{3 2 5 3}$ & $\mathbf{3 0 2 3}$ & $\mathbf{1 0 1 7}$ \\
$\mathbf{C l}^{\mathbf{b}}$ & $\mathbf{0 . 0 0 9 8}$ & $\mathbf{0 . 0 1 0 3}$ & $\mathbf{0 . 0 1 2 7}$ & $\mathbf{0 . 0 1 7 3}$ & $\mathbf{0 . 0 2 2 0}$ & $\mathbf{0 . 0 2 7 4}$ & $\mathbf{0 . 0 4 2 6}$ \\
$\mathbf{C i d}^{\mathbf{c}}$ & $\mathbf{0 . 0 0 4 1}$ & $\mathbf{0 . 0 0 5 2}$ & $\mathbf{0 . 0 0 7 4}$ & $\mathbf{0 . 0 1 1 6}$ & $\mathbf{0 . 0 1 6 6}$ & $\mathbf{0 . 0 2 2 8}$ & $\mathbf{0 . 0 3 7 6}$ \\
\hline
\end{tabular}

${ }^{a}$ Number of observations.

${ }^{\mathrm{b}}$ Concentration index of income-related inequalities in remaining life-years, undiscounted figures.

${ }^{c}$ Concentration index of income-related inequalities in remaining life-years, discounted figures. 
Table 4: Health status (QALY weights) for males in different ages and income deciles.

Income

\section{Remaining Life-Years}

Age groups

Deciles

\begin{tabular}{|c|c|c|c|c|c|c|c|}
\hline INC1 & $\begin{array}{c}20-29 \\
0.939\end{array}$ & $\begin{array}{c}\mathbf{3 0}-\mathbf{3 9} \\
0.935\end{array}$ & $\begin{array}{c}\mathbf{4 0}-\mathbf{4 9} \\
0.910\end{array}$ & $\begin{array}{c}\mathbf{5 0}-\mathbf{5 9} \\
0.895\end{array}$ & $60-69$ & $70-79$ & $80-84$ \\
\hline INC2 & 0.000 & 0935 & & & & 0.005 & 0.581 \\
\hline INC3 & 0.941 & 0.935 & 0.916 & 0.887 & 0.833 & 0.698 & 0.586 \\
\hline & 0.939 & 0.938 & 0.918 & 0.884 & 0.846 & 0.693 & 0.609 \\
\hline & 0.940 & 0.944 & 0.926 & 0.898 & 0.842 & 0.711 & 0.568 \\
\hline INC5 & 0.940 & 0.942 & 0.928 & 0.901 & 0.842 & 0.715 & 0.593 \\
\hline INC6 & 0.943 & 0.947 & 0.935 & 0.905 & 0.854 & 0.712 & 0.582 \\
\hline INC7 & 0.944 & 0.946 & 0.930 & 0.903 & 0.855 & 0.719 & 0.607 \\
\hline INC8 & 0.946 & 0.947 & 0.932 & 0.914 & 0.861 & 0.728 & 0.588 \\
\hline INC9 & 0.945 & 0.946 & 0.936 & 0.923 & 0.868 & 0.735 & 0.618 \\
\hline INC10 & 0.946 & 0.950 & 0.940 & 0.927 & 0.875 & 0.738 & 0.612 \\
\hline$A L L$ & 0.942 & 0.943 & 0.927 & 0.904 & 0.850 & 0.714 & 0.595 \\
\hline $\mathbf{N}^{\mathrm{a}}$ & 4078 & 4487 & 3474 & 2994 & 3053 & 2507 & 729 \\
\hline $\mathrm{Cl}^{\mathrm{b}}$ & 0.0015 & 0.0028 & 0.0052 & 0.0078 & 0.0101 & 0.0126 & 0.0080 \\
\hline$(\mathrm{Cl}-)^{\mathrm{C}}$ & -0.0244 & -0.0457 & -0.0662 & -0.0735 & -0.0571 & -0.0314 & -0.0118 \\
\hline
\end{tabular}


Table 5: Health status (QALY weights) for females in different ages and income deciles.

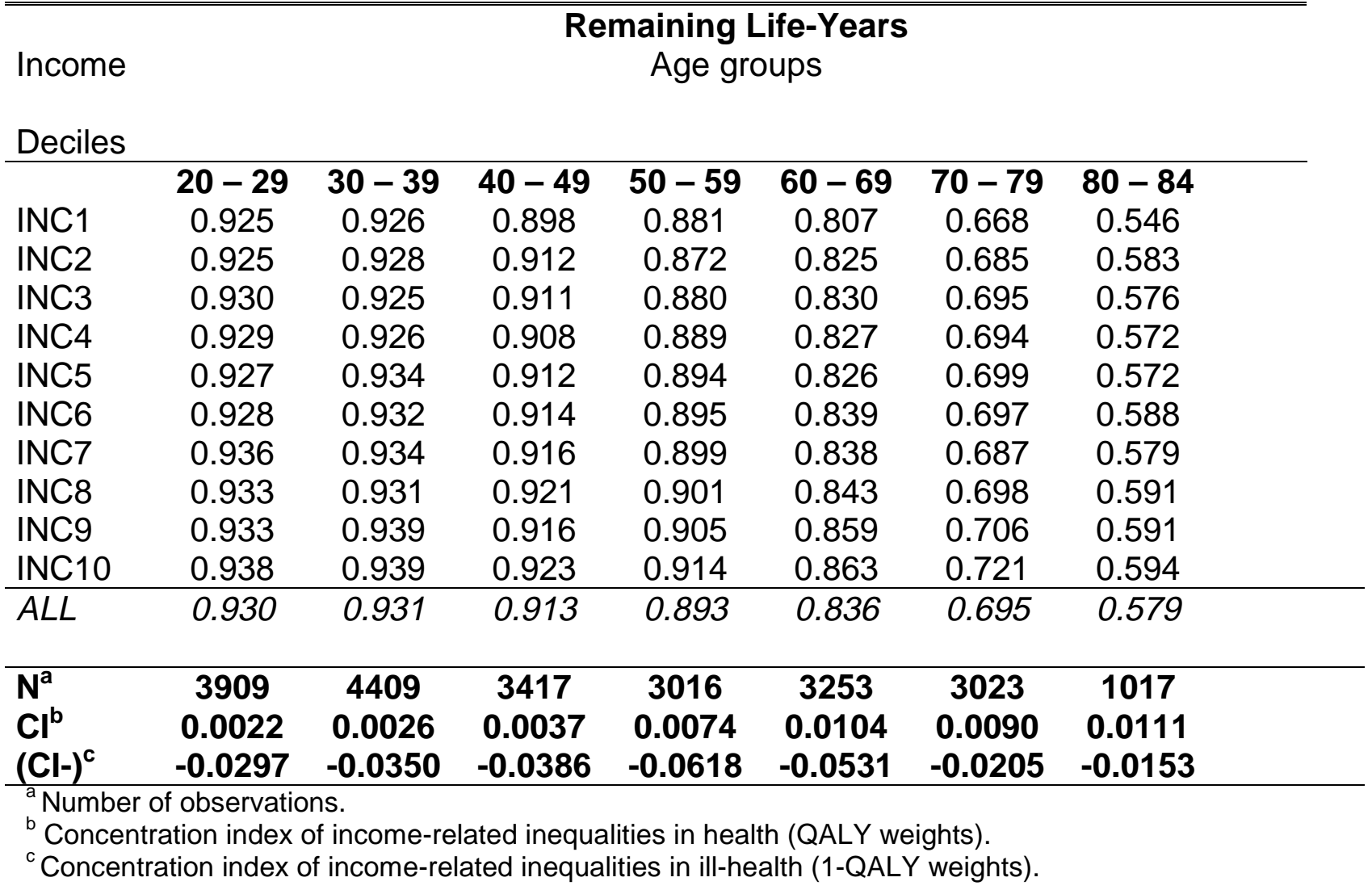


Table 6: Remaining quality-adjusted life-years for males in different ages and income deciles. In parentheses we provide discounted values.

Income

\section{Remaining Life-Years}

Age groups

Deciles

\begin{tabular}{lccccccc}
\hline & $\mathbf{2 0 - 2 9}$ & $\mathbf{3 0 - 3 9}$ & $\mathbf{4 0 - 4 9}$ & $\mathbf{5 0 - 5 9}$ & $\mathbf{6 0 - 6 9}$ & $\mathbf{7 0 - 7 9}$ & $\mathbf{8 0 - 8 4}$ \\
INC1 & 43.663 & 34.372 & 25.413 & 17.359 & 10.300 & 4.720 & 2.299 \\
& $(23.518)$ & $(20.568)$ & $(16.842)$ & $(12.782)$ & $(8.318)$ & $(4.152)$ & $(2.125)$ \\
INC2 & 45.041 & 35.113 & 26.290 & 17.776 & 10.786 & 4.932 & 2.401 \\
& $(23.910)$ & $(20.826)$ & $(17.264)$ & $(12.986)$ & $(8.662)$ & $(4.324)$ & $(2.213)$ \\
INC3 & 45.294 & 35.498 & 26.599 & 17.943 & 11.123 & 4.962 & 2.565 \\
& $(23.944)$ & $(20.988)$ & $(17.412)$ & $(13.060)$ & $(8.907)$ & $(4.344)$ & $(2.359)$ \\
INC4 & 45.576 & 35.926 & 27.037 & 18.425 & 11.181 & 5.175 & 2.410 \\
& $(24.028)$ & $(21.188)$ & $(17.654)$ & $(13.378)$ & $(8.937)$ & $(4.521)$ & $(2.216)$ \\
INC5 & 45.756 & 35.989 & 27.231 & 18.636 & 11.294 & 5.285 & 2.584 \\
& $(24.073)$ & $(21.185)$ & $(17.745)$ & $(13.504)$ & $(9.011)$ & $(4.609)$ & $(2.372)$ \\
INC6 & 46.076 & 36.377 & 27.621 & 18.857 & 11.595 & 5.323 & 2.579 \\
& $(24.192)$ & $(21.365)$ & $(17.958)$ & $(13.637)$ & $(9.230)$ & $(4.636)$ & $(2.364)$ \\
INC7 & 46.322 & 36.524 & 27.602 & 18.934 & 11.724 & 5.459 & 2.771 \\
& $(24.280)$ & $(21.407)$ & $(17.916)$ & $(13.670)$ & $(9.315)$ & $(4.746)$ & $(2.533)$ \\
INC8 & 46.568 & 36.774 & 27.871 & 19.364 & 11.982 & 5.639 & 2.732 \\
& $(24.363)$ & $(21.503)$ & $(18.048)$ & $(13.942)$ & $(9.494)$ & $(4.891)$ & $(2.494)$ \\
INC9 & 46.694 & 36.970 & 28.204 & 19.789 & 12.296 & 5.849 & 3.024 \\
& $(24.378)$ & $(21.551)$ & $(18.212)$ & $(14.204)$ & $(9.709)$ & $(5.056)$ & $(2.749)$ \\
INC10 & 47.229 & 37.796 & 28.904 & 20.383 & 12.923 & 6.308 & 3.320 \\
& $(24.527)$ & $(21.867)$ & $(18.527)$ & $(14.529)$ & $(10.120)$ & $(5.402)$ & $(2.990)$ \\
\hline ALL & 45.822 & 36.134 & 27.277 & 18.746 & 11.520 & 5.365 & 2.669 \\
& $(24.121)$ & $(21.245)$ & $(17.758)$ & $(13.569)$ & $(9.170)$ & $(4.668)$ & $(2.442)$ \\
& & & & & & & \\
\hline $\mathbf{N}^{\mathrm{a}}$ & $\mathbf{4 0 7 8}$ & $\mathbf{4 4 8 7}$ & $\mathbf{3 4 7 4}$ & $\mathbf{2 9 9 4}$ & $\mathbf{3 0 5 3}$ & $\mathbf{2 5 0 7}$ & $\mathbf{7 2 9}$ \\
$\mathbf{C l}^{\mathbf{b}}$ & $\mathbf{0 . 0 1 1 7}$ & $\mathbf{0 . 0 1 4 7}$ & $\mathbf{0 . 0 1 9 7}$ & $\mathbf{0 . 0 2 7 2}$ & $\mathbf{0 . 0 3 5 6}$ & $\mathbf{0 . 0 4 7 3}$ & $\mathbf{0 . 0 5 9 4}$ \\
$\mathbf{C i d}^{\mathbf{c}}$ & $\mathbf{0 . 0 0 6 4}$ & $\mathbf{0 . 0 0 9 6}$ & $\mathbf{0 . 0 1 4 7}$ & $\mathbf{0 . 0 2 2 1}$ & $\mathbf{0 . 0 3 0 8}$ & $\mathbf{0 . 0 4 3 1}$ & $\mathbf{0 . 0 5 5 2}$ \\
\hline
\end{tabular}

${ }^{a}$ Number of observations.

${ }^{\mathrm{b}}$ Concentration index of income-related inequalities in remaining QALYs, undiscounted figures.

${ }^{\mathrm{C}}$ Concentration index of income-related inequalities in remaining QALYs, discounted figures. 
Table 7: Remaining quality-adjusted life-years for females in different ages and income deciles. In parentheses we provide discounted values.

Income

\section{Remaining Life-Years}

Age groups

Deciles

\begin{tabular}{lccccccc}
\hline & $\mathbf{2 0 - 2 9}$ & $\mathbf{3 0 - 3 9}$ & $\mathbf{4 0 - 4 9}$ & $\mathbf{5 0 - 5 9}$ & $\mathbf{6 0 - 6 9}$ & $\mathbf{7 0 - 7 9}$ & $\mathbf{8 0 - 8 4}$ \\
INC1 & 45.685 & 36.489 & 27.328 & 19.180 & 11.750 & 5.660 & 2.854 \\
& $(23.849)$ & $(21.175)$ & $(17.551)$ & $(13.733)$ & $(9.216)$ & $(4.851)$ & $(2.579)$ \\
INC2 & 46.562 & 37.174 & 28.440 & 19.450 & 12.318 & 5.958 & 3.177 \\
& $(24.063)$ & $(21.408)$ & $(18.106)$ & $(13.854)$ & $(9.613)$ & $(5.088)$ & $(2.859)$ \\
INC3 & 47.116 & 37.248 & 28.636 & 19.867 & 12.529 & 6.120 & 3.174 \\
& $(24.267)$ & $(21.408)$ & $(18.185)$ & $(14.341)$ & $(9.755)$ & $(5.218)$ & $(2.854)$ \\
INC4 & 47.256 & 37.434 & 28.641 & 20.275 & 12.571 & 6.161 & 3.180 \\
& $(24.287)$ & $(21.477)$ & $(18.158)$ & $(14.426)$ & $(9.775)$ & $(5.247)$ & $(2.857)$ \\
INC5 & 47.311 & 37.897 & 28.916 & 20.534 & 12.666 & 6.264 & 3.227 \\
& $(24.277)$ & $(21.698)$ & $(18.298)$ & $(14.677)$ & $(9.832)$ & $5.327)$ & $(2.895)$ \\
INC6 & 47.516 & 37.948 & 29.133 & 20.665 & 13.008 & 6.290 & 3.386 \\
& $(24.330)$ & $(21.697)$ & $(18.298)$ & $(14.667)$ & $(10.072)$ & $(5.345)$ & $(3.032)$ \\
INC7 & 47.075 & 38.187 & 29.327 & 20.924 & 13.099 & 6.225 & 3.365 \\
& $(24.570)$ & $(21.795)$ & $(18.298)$ & $(14.854)$ & $(10.127)$ & $(5.287)$ & $(3.010)$ \\
INC8 & 48.090 & 38.182 & 29.693 & 21.106 & 13.335 & 6.439 & 3.517 \\
& $(24.537)$ & $(21.757)$ & $(18.298)$ & $(14.875)$ & $(10.283)$ & $(5.456)$ & $(3.139)$ \\
INC9 & 48.279 & 38.791 & 29.699 & 21.375 & 13.813 & 6.647 & 3.606 \\
& $(24.576)$ & $(22.033)$ & $(18.644)$ & $(15.127)$ & $(10.613)$ & $(5.615)$ & $(3.210)$ \\
INC10 & 49.040 & 39.343 & 30.408 & 22.171 & 14.396 & 7.185 & 3.904 \\
& $(24.817)$ & $(22.200)$ & $(18.968)$ & $(15.400)$ & $(10.971)$ & $(6.019)$ & $(3.448)$ \\
\hline ALL & 47.492 & 37.869 & 29.022 & 20.554 & 12.948 & 6.295 & 3.339 \\
& $(24.357)$ & $(21.665)$ & $(18.349)$ & $(14.439)$ & $(10.026)$ & $(5.345)$ & $(2.988)$ \\
& & & & & & & \\
\hline $\mathbf{N}^{\mathrm{a}}$ & $\mathbf{3 9 0 9}$ & $\mathbf{4 4 0 9}$ & $\mathbf{3 4 1 7}$ & $\mathbf{3 0 1 6}$ & $\mathbf{3 2 5 3}$ & $\mathbf{3 0 2 3}$ & $\mathbf{1 0 1 7}$ \\
$\mathbf{C l}^{\mathbf{b}}$ & $\mathbf{0 . 0 1 0 7}$ & $\mathbf{0 . 0 1 1 9}$ & $\mathbf{0 . 0 1 5 4}$ & $\mathbf{0 . 0 2 4 1}$ & $\mathbf{0 . 0 3 1 6}$ & $\mathbf{0 . 0 3 2 9}$ & $\mathbf{0 . 0 4 6 0}$ \\
$\mathbf{C i d}^{\mathbf{c}}$ & $\mathbf{0 . 0 0 6 1}$ & $\mathbf{0 . 0 0 7 7}$ & $\mathbf{0 . 0 1 1 1}$ & $\mathbf{0 . 0 1 9 3}$ & $\mathbf{0 . 0 2 7 1}$ & $\mathbf{0 . 0 2 9 5}$ & $\mathbf{0 . 0 4 2 5}$ \\
\hline
\end{tabular}

${ }^{a}$ Number of observations.

${ }^{\mathrm{b}}$ Concentration index of income-related inequalities in remaining QALYs, undiscounted figures.

${ }^{\mathrm{C}}$ Concentration index of income-related inequalities in remaining QALYs, discounted figures. 


\section{APPENDIX}

Table A1: Results from the Cox Proportional Hazard Model. Boldface numbers indicate significant results at the 5\% level. Number of observations $=43366$.

\begin{tabular}{lrrr} 
Covariate & Coeff & t-value & p-value \\
\hline BOXINC $(\lambda=0.24)^{\mathrm{a}}$ & $\mathbf{- 0 . 0 1 7 5}$ & $\mathbf{- 1 0 . 9 3 8}$ & $\mathbf{0 . 0 0 0 0}$ \\
MALE & $\mathbf{0 . 5 2 1}$ & $\mathbf{2 3 . 8 9 0}$ & $\mathbf{0 . 0 0 0 0}$ \\
AGE & $\mathbf{0 . 0 8 5 0}$ & $\mathbf{1 2 . 1 4 3}$ & $\mathbf{0 . 0 0 0 0}$ \\
SQUARE OF AGE & $\mathbf{0 . 0 0 0 2}$ & $\mathbf{2 . 7 5 0}$ & $\mathbf{0 . 0 0 0 0}$ \\
\hline -Log-Likelihood value & 81739.56 & & \\
Iterations Completed & 6 & & \\
\hline${ }^{a}$ BOXINC $=$ (annuitized life-time household income per consumption unit $\left.{ }^{\lambda}-1\right) / \lambda$
\end{tabular}

Table A2: Results from the OLS of $\log$ (QALY weight /(1-QALY weight)). Boldface numbers indicate significant results at the 5\% level. Number of observations $=43366$.

\begin{tabular}{lrrr} 
Covariate & Coeff & t-value & p-value \\
\hline CONSTANT & $\mathbf{1 . 4 2 6}$ & $\mathbf{4 9 . 7 6 6}$ & $\mathbf{0 . 0 0 0 0}$ \\
BOXINC $(\lambda=0.35)^{\text {a }}$ & $\mathbf{0 . 0 0 2}$ & $\mathbf{2 1 . 8 2 1}$ & $\mathbf{0 . 0 0 0 0}$ \\
MALE & $\mathbf{0 . 1 6 3}$ & $\mathbf{3 0 . 1 1 1}$ & $\mathbf{0 . 0 0 0 0}$ \\
AGE & $\mathbf{0 . 0 6 0}$ & $\mathbf{6 4 . 8 5 4}$ & $\mathbf{0 . 0 0 0 0}$ \\
SQUARE OF AGE & $\mathbf{- 0 . 0 0 1}$ & $\mathbf{- 1 0 6 . 5 2 0}$ & $\mathbf{0 . 0 0 0 0}$ \\
\hline $\mathrm{R}^{2}$ & 0.642 & & \\
\hline
\end{tabular}

${ }^{\mathrm{a}} \mathrm{BOXINC}=\left(\right.$ annuitized life-time household income per consumption unit $\left.{ }^{\lambda}-1\right) / \lambda$ 